\title{
Variabilidade espacial de variáveis geobiofísicas nas nascentes da bacia hidrográfica do Ribeirão Santa Cruz
}

\author{
Marcelo de C. Alves ${ }^{1}$, Soraya A. Botelho ${ }^{2}$, Lilian V. A. Pinto ${ }^{3}$, Edson A. Pozza ${ }^{4}$, \\ Marcelo S. de 0 liveira ${ }^{5}$, Elizabeth Ferreira ${ }^{1} \&$ Hélcio Andrade ${ }^{6}$
}

\section{RESU M O}

O bjetivou-se, em Lavras, M G, caracterizar a estrutura e a magnitude de dependência espacial de variáveis ambientais nas nascentes da bacia hidrográfica do Ribeirão Santa Cruz, por métodos de interpolação geoestatística. 0 s métodos de interpolação por krigagem ordinária e inverso da potência da distância (IPD) foram utilizados para representar altitude, vazão, áreas de recarga e de vegetação de 175 nascentes perenes em 86,99 km² de área. O bservou-se melhor desempenho da estimação por krigagem quando comparado ao método IPD.

Palavras-chave: geoestatística, krigagem ordinária, inverso da potência da distância, semivariograma

\section{Spatial variability of geobiophysical variables in the springs of the Ribeirão Santa Cruz watershed}

\begin{abstract}
The objective of this study was to characterize the structure and the spatial dependence magnitude of geobiophysical variables in the springs of the Ribeirão Santa Cruz stream watershed, Lavras, M G, using geostatistical interpolation methods. O rdinary kriging system and inverse power distance (IPD) interpolation methods were used to map the altitude, flow, areas of recharge and vegetation of 175 perennial springs in an area of $86.99 \mathrm{~km}^{2}$. Better peformance was observed with estimation using the kriging method as compared to the IPD method.
\end{abstract}

Key words: geostatistics, ordinary kriging, inverse distance weighting, semivariogram

\footnotetext{
1 DEG/U FLA, CP 37, CEP 37200-000, Lavras, MG. Fone: (35) 3829-1494/1481. E-mail: marcelocarvalhoalves@gmail.com; 3829-1462. E-mail: beth@ufla.br

2 DCF/U FLA. Fone: (35) 3829-1422. E-mail: sbotelho@ufla.br

3 EAFI, Visconde de Mauá. Praça Tiradentes 416, centro, CEP 37576-000, Inconfidentes, MG. Fone: (35) 3464-1200. E-mail: lilianvap@gmail.com

${ }^{4}$ DFP/U FLA. Fone: (35) 3829-1791/1289. E-mail: eapozza@ufla.br

5 DEX/U FLA. Fone: (35) 3829-1373. E-mail: marcelo.oliveira@ufla.br

${ }^{6}$ DCS/U FLA. Fone: (35) 3829-1261/1637. E-mail: handrade@ufla.br
} 


\section{INTRODUÇÃO}

A disponibilidade de recursos hídricos é limitada e sua demanda cresceu com o aumento populacional, de forma a determinar disputas pelo direito de uso de quotas de água (Boken et al., 2004). As principais fontes de recursos hídricos disponíveis ao sustento humano não excedem $90.000 \mathrm{~km}^{3}$ ou 0,26\% da água doce da Terra (Shiklomanov, 1998); poderia ser o bastante para uma demanda global de $5.000 \mathrm{~km}^{3}$ por ano, se não fosse considerada a variabilidade da distribuição de água ao longo da superfície terrestre, as enchentes e secas catastróficas em algumas regiões, a degradação dos recursos naturais e as mudanças climáticas globais (Salinger, 2005).

A bacia hidrográfica é a unidade de estudo fundamental para a conservação do meio ambiente, formada por microbacias, as quais possuem pequenos riachos que constituem a malha de drenagem dessa bacia (Alves, 2000). Nessas unidades, a falta de informações necessárias para entender os fenômenos naturais de maneira ampla e integrada, contribui para a ocorrência de desequilíbrios ambientais (Alvarenga \& Paula, 2000; Souza \& Fernandes, 2000) e problemas de conservação do solo e da água (Lombardi Neto, 1992).

Por meio de metodologias de análise baseadas em Sistema de Informações Geográficas (SIG) e Geoestatística, foi demonstrada a possibilidade de otimização da preservação ambiental, caracterização do meio físico, o uso e o manejo dos recursos naturais de bacias hidrográficas considerandose a variabilidade espacial de atributos geobiofísicos (Boken et al., 2004; Pinto et al., 2004; Vieira et al., 1992). Para representar os valores da variável estudada em áreas não amostradas, foram utilizados métodos de interpolação dos dados com diferenças quanto à qualidade dos resultados (Burrough \& McDonell, 1998; Isaaks \& Srivastava, 1989; Carvalho \& Vieira, 2001).

A escolha do melhor método de interpolação pode variar de acordo com a natureza dos dados (contínuos ou discretos), número de amostras, arranjo utilizado, distância entre amostras, variabilidade e dependência espacial do atributo estudado, informação espacial fornecida pelo método, tempo demandado pela análise, nível teórico do analista, processamento computacional, acurácia e precisão da estimativa, entre outros fatores (Andriotti, 2003; Burrough \& McDonell, 1998; Kravchenko, 2003; Kravchenko \& Bullock, 1999; Mueller et al., 2001; Zimmerman et al., 1999).

A variedade de interpoladores existentes gera dúvidas sobre qual método é mais apropriado, de acordo com cada contexto específico (Zimmerman et al., 1999). Estimativas acuradas de variáveis ambientais foram obtidas por meio dos métodos de interpolação pelo inverso da potência da distância (IPD) e por krigagem (Boken et al., 2004; Kravchenko, 2003; Musa et al., 2002). Esses métodos apresentaram a vantagem de estimar valores baseados em locais não amostrados, atribuindo pesos diferenciados segundo a localização da amostra e sua vizinhança (Mueller et al., 2001; Kravchenko \& Bullock, 1999). Com a metodologia da krigagem ordinária em blocos, é possível proceder a uma interpolação, de forma não tendenciosa e com minimização de variância (Isaaks \& Srivastava, 1989), determinar o comportamento da variabilidade em diferentes direções e caracterizar a estrutura e magnitude de dependência espacial de uma variável (Burrough \& McDonell, 1998); já o método do IPD não apresenta as vantagens estatísticas da krigagem; contudo, é menos laborioso, demanda menos tempo de análise e processamento computacional (Mueller et al., 2001; Kravchenko, 2003).

Há muita controvérsia sobre resultados dos trabalhos nos quais se aborda a comparação entre métodos de interpolação (Kravchenko, 2003). Em alguns estudos se constatou maior acurácia da interpolação por IPD que a krigagem (Musa et al., 2002), enquanto em outros se verificou melhor desempenho da krigagem (Kravchenko \& Bullock, 1999; Zimmerman et al., 1999) ou estimativas muito próximas entre os dois métodos, apesar da superioridade da krigagem (Conde \& Yamamoto, 1996; Kravchenko, 2003). Wollenhaupt et al. (1994) compararam o IPD com a krigagem no mapeamento de níveis de $\mathrm{P}$ e $\mathrm{K}$ em solos e verificaram maior acurácia do método IPD; entretanto, Kravchenko \& Bullock (1999), estudando a interpolação por krigagem ordinária e IPD no mapeamento de propriedades de solo constataram, na maioria das situações, melhor desempenho da krigagem. Kravchenko (2003) também verificou melhor desempenho da krigagem com relação ao IPD no mapeamento de propriedades de solo; porém, quando não foi possível obter parâmetros confiáveis nos semivariogramas, a krigagem apresentou menor acurácia.

Segundo Kravchenko (2003) e Mueller et al. (2001), em situações com menor densidade de amostragem e maior distância entre os pontos da malha amostral, o alcance da correlação espacial da variável estudada pode ser excedido de forma a dificultar sua predição espacial com acurácia e precisão satisfatória, por não captar as características estruturais das variáveis estudadas.

No intuito de contribuir com metodologias para solucionar problemas de disputas de água e avaliar a interação de variáveis ambientais com os recursos hídricos de determinada região, objetivou-se caracterizar a estrutura e magnitude de dependência espacial das variáveis altitude, vazão, vegetação nativa e área de recarga das nascentes da bacia do Ribeirão Santa Cruz, Lavras, MG, por meio das metodologias de análise geoestatística de krigagem ordinária e do inverso da potência da distância.

\section{MATERIAL E MÉTODOS}

\section{Área de estudo}

A bacia hidrográfica do Ribeirão Santa Cruz está localizada entre as coordenadas $21^{\circ} 09^{\prime} 39^{\prime \prime}$ e $21^{\circ} 20^{\prime} 14^{\prime \prime}$ de latitude sul e $44^{\circ} 51^{\prime} 36^{\prime \prime}$ e $45^{\circ} 00^{\prime} 00^{\prime \prime}$ de longitude oeste de Greenwich, com área de $86,99 \mathrm{~km}^{2}$, perímetro de $62,49 \mathrm{~km}$, correspondente a $15,44 \%$ da área do município de Lavras. A bacia hidrográfica estudada pertence à do Rio Grande e seu curso d'água principal é representado pelo Ribeirão Santa Cruz, com extensão de 30,09 km. O Ribeirão Santa Cruz é 
tributário do Rio Capivari que, por sua vez, o é também do Rio Grande, e este, integrante da bacia do Rio Paraná (Pinto, 2003).

Obtenção dos dados planialtimétricos e de localização das nascentes

Para obter os dados planialtimétricos, compilaram-se informações cartográficas das cartas do IBGE, folha de Lavras (SF-23-X-C-I-1) e Itumirim (SF-23-X-C-I-3), escala 1:50.000, ano de 1975. A delimitação da bacia foi determinada com base nas linhas de cumeadas das curvas de nível de cartas topográficas do IBGE, com eqüidistância de $20 \mathrm{~m}$.

Para localizar as nascentes foi considerado cada início dos tributários do Ribeirão Santa Cruz, pela rede de drenagem da carta do IBGE, a partir da qual se localizaram as 175 nascentes perenes, com um receptor GPS ('Global Position System'), modelo 'Garmim-3Plus'.

Obtenção dos dados de vegetação nativa no entorno da nascente

A vegetação no entorno das nascentes foi medida com uma trena até o raio de 50 metros; mediu-se a vegetação acima (R1), abaixo (R2), à direita (R3) e à esquerda (R4) da nascente; a margem direita e a esquerda foram orientadas pelo sentido do escoamento do leito do curso principal, conforme descrito por Pinto (2003).

\section{Obtenção dos dados da área de recarga das nascentes}

Obtiveram-se os dados da área de recarga com base em fotos aéreas verticais tomadas pela AEROSUL S.A. (Fotogrametria Sul do Brasil) e AERODATA (Aerofotogrametria e Consultoria S.A.) para o IBC-GERCA (Instituto Brasileiro do Café - Grupo Executivo de Racionalização da Cafeicultura) no ano de 1979, com escala aproximada de 1:25.000 e formato $23 \times 23 \mathrm{~cm}$. Para o recobrimento das áreas de recarga da bacia hidrográfica do Ribeirão Santa Cruz, foram utilizadas 17 fotos da folha do foto índice SF 23-X-C-I. As fotografias foram interpretadas com o auxílio de um estereoscópio de bolso, o qual fornece uma visão tridimensional do terreno, com base na metodologia de Rocha (1985) e Pinto (2003).

\section{Obtenção dos dados de vazão das nascentes}

As aferições hidrológicas foram realizadas em outubro de 2002, mês com menores vazões históricas; a vazão $\left(\mathrm{m}^{3} \mathrm{~s}^{-1}\right)$ foi quantificada com base em medições realizadas nas nascentes, pelo processo direto. Em situações em que não foi possível coletar a água por um recipiente, utilizaram-se medidores de vazão sob regime crítico, modelo WSC Flume (Bernardo, 1989), instalados em nível com o solo. Após um período de estabilização da água sobre a calha realizou-se a aferição da vazão no ponto marcado da calha, com o auxílio de uma régua, conforme descrito em Pinto et al. (2004).

\section{Métodos de espacialização dos dados}

Utilizaram-se os métodos de interpolação pelo inverso da potência da distância e de krigagem ordinária, os quais serão abordados a seguir.

\section{Inverso da potência da distância (IPD)}

O método IPD foi utilizado para espacializar as variáveis: curva de nível, área de recarga, área de vegetação nativa e vazão. As estimativas do método IPD são influenciadas pelos valores da vizinhança, embora os pesos sejam determinados apenas em função da distância a partir do local da interpolação; para tal, pressupõe-se que observações próximas tendem a ser semelhantes. $O$ valor da variável $Z$ em locais não amostrados $\mathrm{x}_{0}$, é estimado por $\mathrm{Z}^{*}\left(\mathrm{x}_{0}\right)$ com base nos dados da vizinhança $\mathrm{Z}\left(\mathrm{x}_{\mathrm{i}}\right)$, com a equação de (Isaaks \& Srivastava, 1989):

$$
\mathrm{Z} *\left(\mathrm{x}_{\mathrm{o}}\right)=\frac{\sum_{\mathrm{i}=1}^{\mathrm{n}} \mathrm{w}_{\mathrm{i}} \mathrm{Z}\left(\mathrm{x}_{\mathrm{i}}\right)}{\sum_{\mathrm{i}=1}^{\mathrm{n}} \mathrm{w}_{\mathrm{i}}}
$$

em que,

$\mathrm{w}_{\mathrm{i}}$ são os pesos designados a cada valor de $\mathrm{Z}\left(\mathrm{x}_{\mathrm{i}}\right)$ e $\mathrm{n}$ é o número de vizinhos mais próximos amostrados utilizados no cálculo da estimativa. Os pesos para a estimativa do IPD foram calculados por (Isaaks \& Srivastava, 1989):

$$
\mathrm{w}_{\mathrm{i}}=\frac{1}{\mathrm{~d}_{\mathrm{i}}^{\mathrm{p}}}
$$

em que:

$\mathrm{d}_{1}, \ldots, \mathrm{d}_{\mathrm{n}}$ são as distâncias de cada $n$ amostras até o ponto a ser estimado e p é o expoente. A mudança no expoente $p$ alterou a qualidade da estimativa em outros estudos (Isaaks \& Srivastava, 1989; Weber \& Englund, 1994), de forma que baixos valores da potência tenderam a suavizar os valores extremos, enquanto valores elevados o realçaram (Conde \& Yamamoto, 1996).

O número de vizinhos foi escolhido em função da auto-validação. Utilizou-se a potência de dois no cálculo das estimativas.

\section{Krigagem ordinária}

Na Geoestatística, a análise do modelo de semivariograma é uma etapa importante visto que o modelo escolhido é a interpretação da estrutura de correlação espacial a ser utilizada nos procedimentos inferenciais da krigagem (Camargo, 1997). O modelo relaciona a semivariância com o vetor distância (Burrough \& McDonnell, 1998):

$$
\hat{\gamma}(\mathrm{h})=\frac{1}{2 \mathrm{~N}(\mathrm{~h})} \sum_{\mathrm{i}=1}^{\mathrm{N}(\mathrm{h})}\left[\mathrm{Z}\left(\mathrm{x}_{\mathrm{i}}\right)-\mathrm{Z}\left(\mathrm{x}_{\mathrm{i}}+\mathrm{h}\right)\right]^{2}
$$

em que:

$\hat{\gamma}(\mathrm{h})$ - Semivariância estimada

$\mathrm{N}(\mathrm{h})$ - Número de pares de observações $\mathrm{Z}\left(\mathrm{x}_{\mathrm{i}}\right), \mathrm{Z}\left(\mathrm{x}_{\mathrm{i}}+\mathrm{h}\right)$, separados pela distância $h$.

Compreende-se, pela análise, o levantamento do semivariograma experimental e, posteriormente, o ajuste a uma família de modelos teóricos. É importante representar, no modelo ajustado, a tendência de $\hat{\gamma}(\mathrm{h})$ em relação a $\mathrm{h}$, de forma que as estimativas obtidas baseadas na krigagem sejam mais exatas e confiáveis (Camargo, 1997). 
Os modelos teóricos de semivariogramas isotrópicos foram ajustados por (Olea, 1999):

$$
\begin{aligned}
& \text { Modelo Esférico: } \gamma(\mathrm{h})=\left\{\begin{array}{l}
\mathrm{C}\left(\frac{3}{2} \frac{\mathrm{h}}{\mathrm{a}}-\frac{1}{2}\left(\frac{\mathrm{h}}{\mathrm{a}}\right)^{3}\right), 0 \leq \mathrm{h}<\mathrm{a} \\
\mathrm{C} \quad, \mathrm{h} \geq \mathrm{a}
\end{array}\right. \\
& \text { Modelo Exponencial: } \gamma(\mathrm{h})=\mathrm{C}\left(1-\mathrm{e}^{-\frac{3 \mathrm{~h}}{\mathrm{a}}}\right)
\end{aligned}
$$

em que:

C é o patamar, a, o alcance e $\mathrm{h}$, a distância.

O modelo de melhor ajuste foi escolhido em função dos parâmetros dos semivariogramas, ou seja, o maior coeficiente de determinação $\left(\mathrm{R}^{2}\right)$, a menor soma de quadrados do resíduo e o menor erro de predição da auto-validação ('crossvalidation "). Para a análise do grau de dependência espacial das variáveis utilizou-se a classificação adaptada de Cambardella et al. (1994) considerando-se, de forte dependência espacial, a relação entre a contribuição $\left(\mathrm{C}_{1}\right)$ e o patamar $(\mathrm{C})$ do semivariograma teórico $\leq 0,25$, moderada quando entre 0,25 e 0,75 e de fraca quando $>0,75$.

Após o ajuste dos semivariogramas utilizou-se a interpolação dos dados pelo método da krigagem ordinária em blocos, para espacializar as variáveis curvas de nível, área de recarga, área de vegetação nativa e vazão. A estimação do número de vizinhos foi escolhida em função da auto-validação.

Em formato matricial, as equações da krigagem ordinária em blocos foram estimadas por (Webster \& Oliver, 2001):

$$
\mathrm{A} \lambda=\mathrm{b} \approx \mathrm{A}^{-1} \mathrm{~b}=\left[\begin{array}{l}
\lambda \\
\varphi
\end{array}\right]
$$

em que:

A é a matriz de semivariância entre os pares de dados, b é o vetor de semivariâncias entre cada par e a estimativa do bloco e $\lambda$ é o vetor de pesos.

$$
\mathrm{A}=\left[\begin{array}{ccccc}
\gamma\left(\mathrm{x}_{1}, \mathrm{x}_{1}\right) & \gamma\left(\mathrm{x}_{1}, \mathrm{x}_{2}\right) & \ldots & \gamma\left(\mathrm{x}_{1}, \mathrm{x}_{\mathrm{n}}\right) & 1 \\
\gamma\left(\mathrm{x}_{2}, \mathrm{x}_{1}\right) & \gamma\left(\mathrm{x}_{2}, \mathrm{x}_{2}\right) & \ldots & \gamma\left(\mathrm{x}_{2}, \mathrm{x}_{\mathrm{n}}\right) & 1 \\
\cdot & \cdot & \cdot & \cdot & \cdot \\
\cdot & \cdot & \cdot & . & \cdot \\
\cdot & \cdot & \cdot & \cdot & \cdot \\
\gamma\left(\mathrm{x}_{\mathrm{n}}, \mathrm{x}_{1}\right) & \gamma\left(\mathrm{x}_{\mathrm{n}}, \mathrm{x}_{2}\right) & \ldots & \gamma\left(\mathrm{x}_{\mathrm{n}}, \mathrm{x}_{\mathrm{n}}\right) & 1 \\
1 & 1 & \ldots & 1 & 0
\end{array}\right]
$$

$$
\lambda=\left[\begin{array}{c}
\lambda_{1} \\
\lambda_{2} \\
\cdot \\
\cdot \\
\cdot \\
\lambda_{\mathrm{n}} \\
\varphi
\end{array}\right]
$$

$$
\mathrm{b}=\left[\begin{array}{c}
\bar{\gamma}\left(\mathrm{x}_{1}, \mathrm{~B}\right) \\
\bar{\gamma}\left(\mathrm{x}_{2}, \mathrm{~B}\right) \\
\cdot \\
\cdot \\
\bar{\gamma}\left(\mathrm{x}_{\mathrm{n}}, \mathrm{B}\right) \\
1
\end{array}\right]
$$

Preferiu-se a krigagem ordinária em blocos, por resultar em superfície mais suavizada quando comparada com a krigagem ordinária pontual (Burrough \& McDonnell, 1998).

Em formato matricial a variância da krigagem $\left(\hat{\sigma}^{2}\right)$ foi estimada por:

$$
\hat{\sigma}^{2}(B)=b^{T} \lambda-\bar{\gamma}(B, B)
$$

em que:

$\mathrm{b}^{\mathrm{T}}$ é o transposto do vetor $\mathrm{b}$.

As estimativas de krigagem e IPD foram sobrepostas ao modelo de elevação digital da área experimental pelo método de sobreposição ponderada em SIG (Surfer, 2002).

\section{Comparação entre os métodos de interpolação por IPD e krigagem}

A qualidade das estimativas das variáveis curvas de nível, área de recarga, área de vegetação natural e vazão das nascentes perenes, foi mensurada de forma a se conhecer a incerteza da representação das variáveis analisadas.

O critério de informação Akaike (Â), baseado na teoria da decisão, foi utilizado para averiguar o desempenho comparativo dos métodos de interpolação por IPD e krigagem, de acordo com a equação (Webster \& Oliver, 2001):

$$
\widehat{\mathrm{A}}=2 \mathrm{p}+\mathrm{nln}\left(\frac{\mathrm{RSS}}{\mathrm{n}}\right)
$$

em que:

n é o numero de observações, ln é o logaritmo natural, RSS é a soma de quadrados de resíduos e p é o número de parâmetros do modelo. Considerou-se a metodologia mais satisfatória àquela com menores valores de AIC.

O quadrado médio do erro (QMErro) da predição foi utilizado para avaliar a acurácia das estimativas de acordo com a equação (Webster \& Oliver, 2001):

$$
\text { QMErro }=\sqrt{\frac{\sum_{\mathrm{i}=1}^{\mathrm{n}}\left(\hat{\mathrm{Z}}\left(\mathrm{x}_{\mathrm{i}}\right)-\mathrm{z}\left(\mathrm{x}_{\mathrm{i}}\right)\right)^{2}}{\mathrm{n}}}
$$

em que:

$\mathrm{Z}\left(\mathrm{x}_{\mathrm{i}}\right)$ é o valor estimado por IPD ou krigagem e $\mathrm{z}\left(\mathrm{x}_{\mathrm{i}}\right)$ é o valor observado. Considerou-se como metodologia mais acurada àquela com menores valores de QMErro.

Posteriormente, histogramas com distribuição de frequiências com oito classes foram utilizados para comparar os valores observados e estimados com os métodos de IPD e krigagem, de acordo com a equação de Sturges (Andriotti, 2003): 
$\mathrm{K}=1+3,3 \log _{10} \mathrm{n}$

em que:

$\mathrm{K}$ é o número de intervalos de classe a se dividir os dados amostrais e $\mathrm{n}$ a quantidade de observações, $\mathrm{n}=175$.

\section{Programas utilizados}

O programa $\mathrm{GS}^{+\circledR}$ (Robertson, 1998) foi utilizado para gerar e ajustar os semivariogramas, interpolação e validar os dados. Empregou-se o sistema de mapeamento de superfícies Surfer ${ }^{\circledR}$ (Surfer, 2002) para gerar as superfícies interpoladas por krigagem e IPD.

\section{RESULTADOS E DISCUSSÃO}

A partir da distribuição das observações na bacia hidrográfica do Ribeirão Santa Cruz, constatou-se não haver problemas com escassez de locais amostrados na área, principalmente no caso das amostras das curvas de nível. Em se tratando das observações das nascentes verificou-se, em algumas regiões, excesso, e em outras, deficiência de observações devido à proximidade das nascentes e ao regime temporário do fluxo de água ou desaparecimento de nascentes, respectivamente. A localização das nascentes também foi condizente com a localização da rede de drenagem da área (Figura 1).

Determinou-se a estrutura e a magnitude da dependência espacial dos atributos da bacia hidrográfica do Ribeirão Santa
Cruz, por meio de modelos de semivariogramas isotrópicos teóricos ajustados aos semivariogramas experimentais, com forte estrutura de dependência espacial, de acordo com a classificação adaptada de Cambardella et al. (1994). O melhor ajuste aos dados de curvas de nível foi obtido pelo modelo esférico, com alcance prático de $753 \mathrm{~m}$, provavelmente influenciado pela predominância de relevo ondulado, forteondulado e montanhoso da bacia hidrográfica. Com relação aos dados de vegetação nativa, observou-se alcance prático de $210 \mathrm{~m}$, com o ajuste de um modelo esférico, provavelmente influenciado pela preservação da vegetação ao redor das nascentes e pela necessidade de reflorestamento das áreas de preservação permanente na bacia hidrográfica, conforme relatado por Pinto et al. (2005). Já para vazão das nascentes perenes, ajustou-se o modelo exponencial, com alcance de 1.620 m (Tabela 1) e para os dados relativos à área de recarga, ajustou-se modelo esférico, com alcance prático de $1.610 \mathrm{~m}$.

Tabela 1. Coeficientes e parâmetros de semivariogramas isotrópicos dos atributos curvas de nível (C. nível) (m), área de recarga $\left(\mathrm{m}^{2}\right)$, área de vegetação nativa $\left(\mathrm{m}^{2}\right)$ e vazão das nascentes perenes $\left(\mathrm{L} \mathrm{min}^{-1}\right)$ da bacia hidrográfica do Ribeirão Santa Cruz, Lavras, MG

\begin{tabular}{lccccccc}
\hline & \multirow{3}{*}{ Atributo } & Modelo & \multicolumn{3}{c}{ Parâmetros } & \multicolumn{2}{c}{ Coeficientes } \\
\cline { 3 - 5 } & & $\begin{array}{c}\text { Efeito } \\
\text { pepita (Co) }\end{array}$ & $\begin{array}{c}\text { Patamar } \\
\text { (c) }\end{array}$ & $\begin{array}{c}\text { Alcance } \\
\text { prático (a) }\end{array}$ & $\mathbf{R}^{\mathbf{2}}$ & $\mathbf{C}_{\mathbf{C}} \mathbf{C}_{\mathbf{1}}$ \\
\hline C. nível & Esférico & 1,00 & 1259,00 & 753,00 & 0,97 & 0,99 \\
Recarga & Esférico & $13,00 \mathrm{E}^{8}$ & $54,81 \mathrm{E}^{8}$ & 1610,00 & 0,80 & 0,75 \\
Vegetação & Esférico & 1,00 & 701,40 & 210,00 & 0,92 & 0,99 \\
Vazão & Exponencial & 0,55 & 3,66 & 1620,00 & 0,84 & 0,84 \\
\hline
\end{tabular}

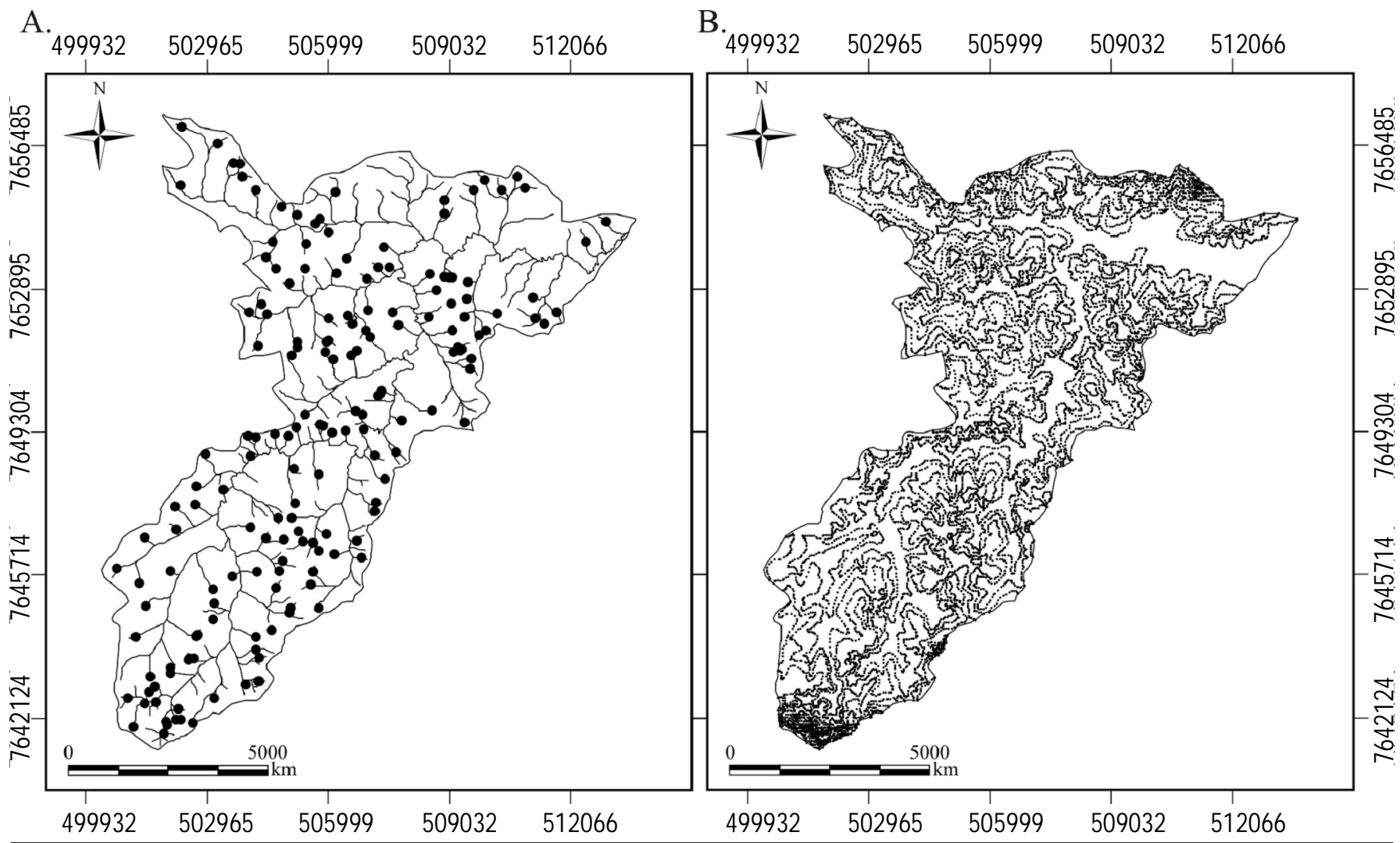

Figura 1. Representações bidimensionais da localização das amostras de vazão, área de recarga, área de vegetação nativa das nascentes, sobre a rede de drenagem (A) e amostras das curvas de nível (B) utilizadas no estudo das variáveis ambientais da bacia hidrográfica do Ribeirão Santa Cruz, Lavras, M G 
A semelhança entre a magnitude dos alcances da vazão das nascentes e das áreas de recarga pode estar relacionada à influência das áreas de recarga no armazenamento da água subterrânea, no regime das nascentes e dos cursos d'água da bacia hidrográfica, conforme relatado por Pinto et al. (2004); de forma semelhante, Desbarats et al. (2002) estudando a altura de colunas de poços de água do aqüífero 'Oak Ridges Moraine', em Ontário, Canadá, em uma área de 250 km², também obtiveram ajuste de semivariogramas com alcance próximo a $2000 \mathrm{~m}$.

Portanto, no presente estudo, de acordo com o alcance obtido pelo semivariograma dos dados de vazão das nascentes e da área de recarga das nascentes da bacia hidrográfica do Ribeirão Santa Cruz, infere-se que a preservação de uma nascente, na região estudada, pode ser influenciada pela vizinhança em um raio próximo a 1.620 m (Tabela 1 e Figura 2).

Ressalta-se que os semivariogramas experimentais apresentaram estrutura de dependência espacial bem definida, caracterizada pelo melhor ajuste dos modelos teóricos ao comportamento da semivariância com relação à distância (Figura 2), conferindo estimativas mais exatas e confiáveis da krigagem quando comparadas às do IPD, com base no critério de Akaike e no quadrado médio do erro das estimativas (Tabela 2).

Tabela 2. Coeficientes de Akaike (Â) e quadrado médio do erro (Q M Erro) das variáveis estimadas na bacia hidrográfica do Ribeirão Santa Cruz, Lavras, M G, pelo método da krigagem ordinária e por inverso da potência da distância (IPD), referentes à: curvas de nível da área $(\mathrm{m})$, área de recarga $\left(\mathrm{m}^{2}\right)$, área de vegetação nativa $\left(\mathrm{m}^{2}\right)$ e vazão das nascentes perenes $\left(\mathrm{L} \mathrm{min}^{-1}\right)$

\begin{tabular}{|c|c|c|c|c|}
\hline \multirow{2}{*}{ Atributo } & \multicolumn{2}{|c|}{ Krigagem } & \multicolumn{2}{|c|}{ IPD } \\
\hline & $\hat{\mathbf{A}}$ & QMErro & $\hat{\mathbf{A}}$ & QMErro \\
\hline C. nível & 1076,35 & $6,44 \mathrm{E}^{9}$ & 1338,71 & $1,29 \mathrm{E}^{11}$ \\
\hline Recarga & 3918,01 & $4,67 \mathrm{E}^{21}$ & 3925,52 & $5,09 \mathrm{E}^{21}$ \\
\hline Vegetação & 2746,75 & $7,18 \mathrm{E}^{15}$ & 2767,38 & $9,09 \mathrm{E}^{15}$ \\
\hline Vazão & 986,21 & $1,31 \mathrm{E}^{7}$ & 989,65 & $1,37 \mathrm{E}^{7}$ \\
\hline
\end{tabular}

Com base nos gráficos de distribuição de freqüência das variáveis observadas e estimadas nos dois métodos, para os dados de curva de nível e área de recarga, verificaram-se valores observados e preditos com distribuições semelhantes, pois a altitude e o relevo da bacia hidrográfica foram submetidos a processos similares de erosão e formação do solo, de modo a conferir estacionariedade de segunda ordem a essas variáveis; constataram-se, para vazão e vegetação nativa, suavização das estimativas, de acordo com a proporção da variabilidade total da amostra, de forma a anular as concentrações localizadas de observações. Tal fato pode ser explicado pelo uso da terra em algumas localidades da bacia hidrográfica, de forma a determinar a modificação dos fatores controladores do comportamento dessas variáveis, acarretando na heterogeneidade acentuada entre certas zonas da área estudada (Figura 3).

De acordo com os mapas confeccionados, observou-se semelhança das áreas mapeadas com os métodos de krigagem e de IPD, porém no método do IPD houve formação de
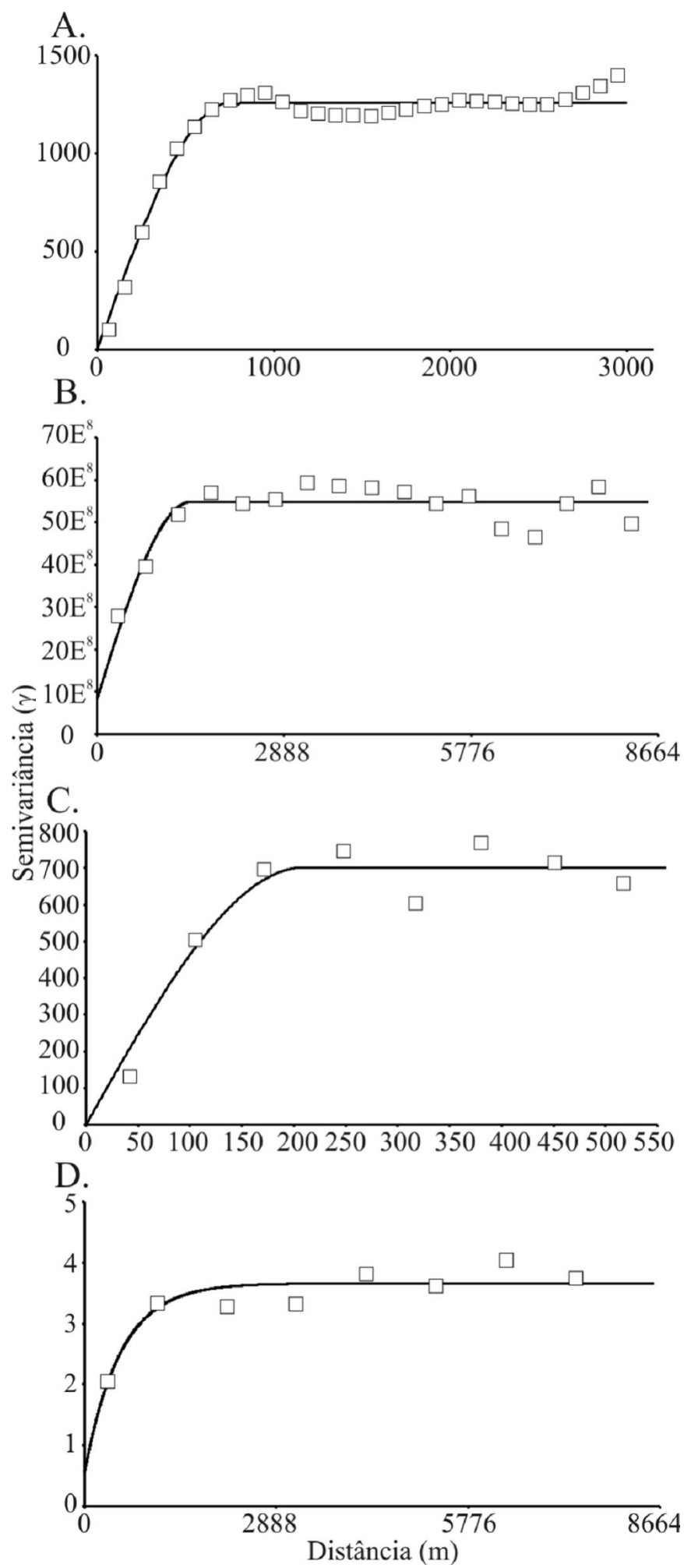

Figura 2. Semivariogramas experimentais e teóricos das variáveis curvas de nível da área $(A)$, área de recarga $\left(\mathrm{m}^{2}\right)(B)$, área de vegetação nativa $\left(\mathrm{m}^{2}\right)$ (C) e vazão (L min-1) (D) da bacia hidrográfica do Ribeirão Santa Cruz, Lavras, MG

contornos concêntricos ao redor dos pontos de amostragem (Andriotti, 2003); já a krigagem apresentou variabilidade espacial diferenciada, definida pelo ajuste dos semivariogramas (Figura 4). Por meio da sobreposição ponderada da recarga, da vegetação e da vazão sobre o modelo de elevação digital 


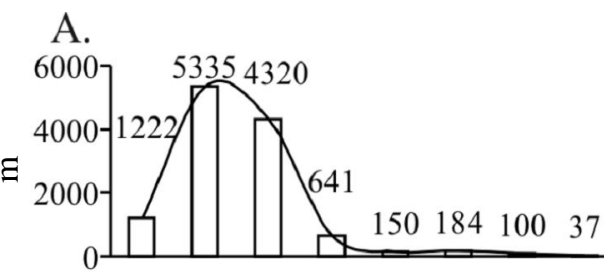

B.

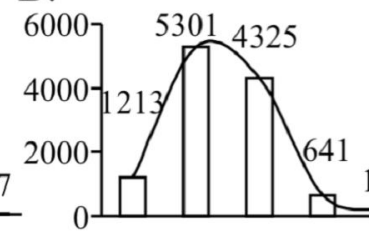

C.

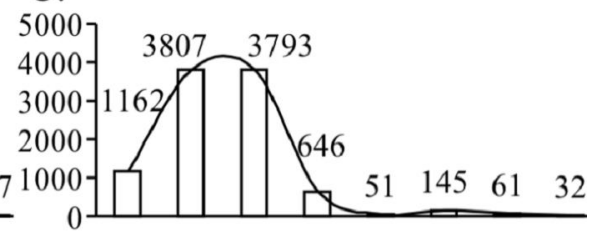
199 \begin{tabular}{lll}
$178 \quad 95 \quad 37$ & 1000 \\
\hline
\end{tabular}

D.

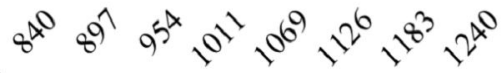

E.

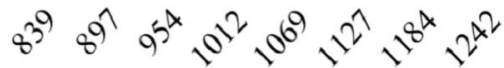

F.

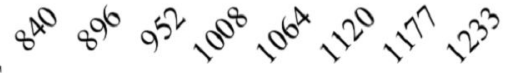

ह $\left.\begin{array}{r}40 \\ 20 \\ 0\end{array}\right]$

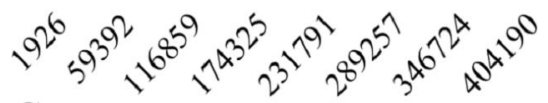

G.
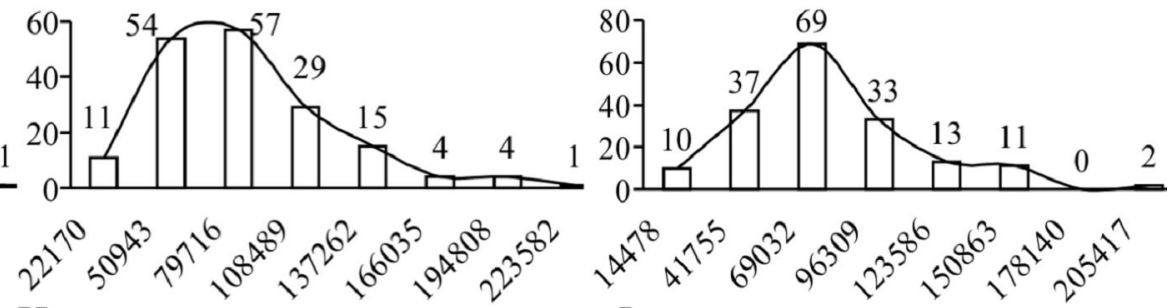

H.
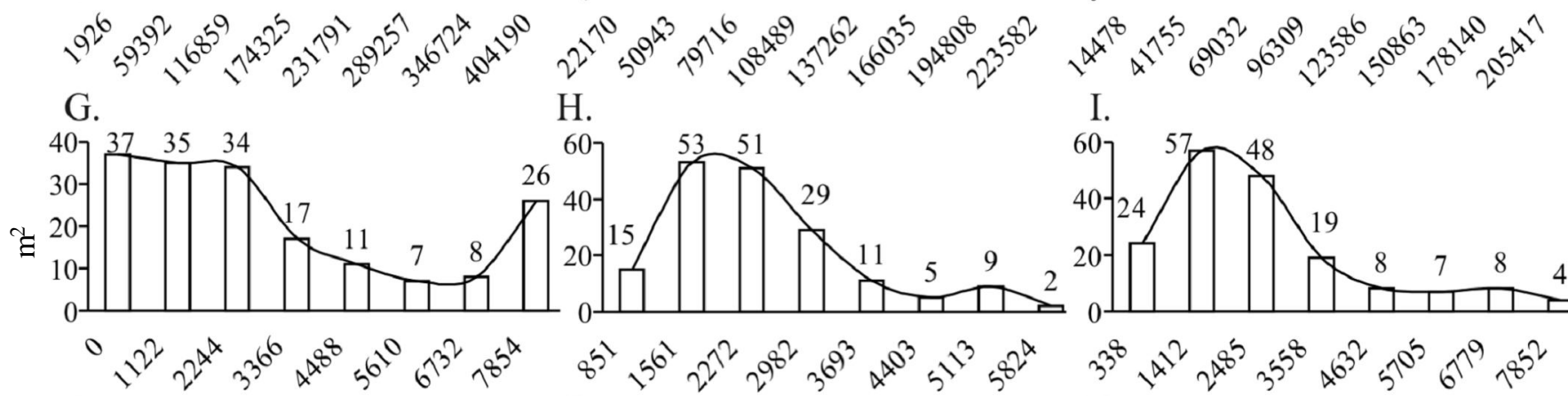

$\mathrm{J}$.

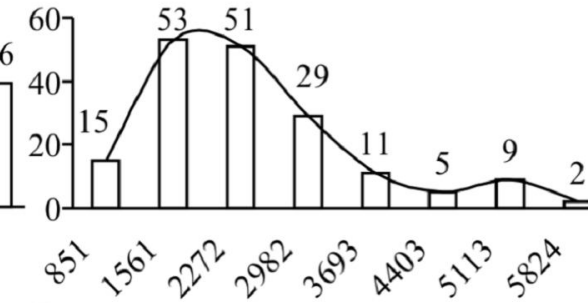

I.
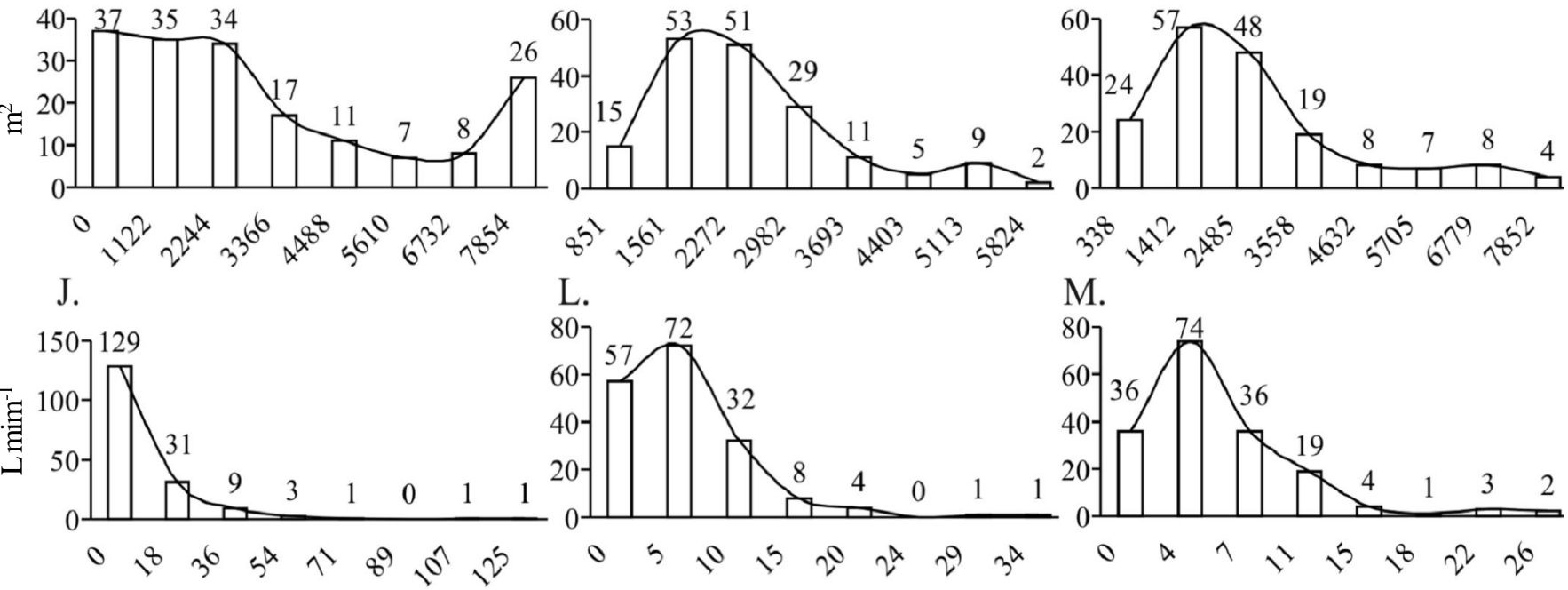

$\mathrm{L}$.

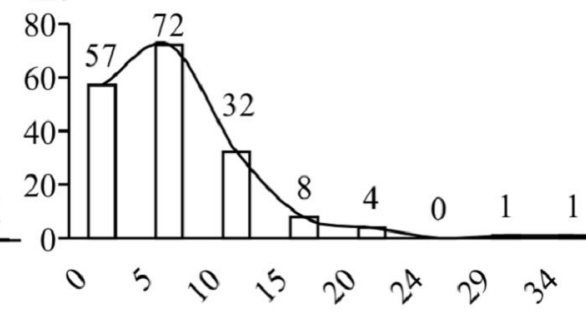

M.

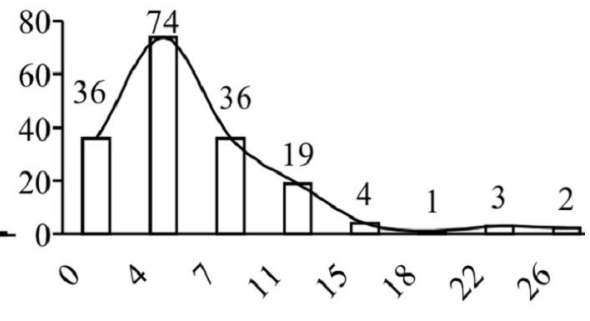

Figura 3. H istogramas de freqüência das variáveis observadas $(A, D, G, J)$ e estimadas pelo método da krigagem $(B, E, H, L)$ e por IPD $(C, F, I, M)$, referentes às curvas de nível da área $(A, B, C)$, área de recarga $\left(m^{2}\right)(D, E, F)$, área de vegetação nativa $\left(m^{2}\right)(G, H, I)$ e vazão $(J, L, M)$ das nascentes perenes $\left(L\right.$ min $\left.^{-1}\right)$ da bacia hidrográfica do Ribeirão Santa Cruz, Lavras, MG

da área, foi possível constatar regiões com altas vazões próximas a regiões de maior altitude (Figura 5). Desbarats et al. (2002) também verificaram, em seu estudo, maiores alturas de coluna de água em áreas de topografia mais elevada. Segundo Pinto (2003), nessas áreas predominam relevo ondulado, forte-ondulado e montanhoso, onde se localizam $53,11 \%$ das nascentes da bacia hidrográfica do Ribeirão Santa Cruz, classificadas como pontuais, já que as características de relevo possibilitam o encontro da camada impermeável do solo com a encosta, originando as nascentes. De acordo com os mesmos autores, os maiores valores de vazão da bacia hidrográfica ocorreram nessas áreas, em função da maior preservação, em virtude do relevo forte ondulado a montanhoso, limitante ao uso de máquinas agrícolas. Essas unidades geomórficas de relevo são representadas, na região de Lavras, pelas classes solos B texturais, Cambissolos e Litólicos (Andrade et al., 1998), as quais possibilitam o armazenamento de água por retenção nos poros capilares do solo e no interior de rochas porosas ou entre fraturas de rochas maciças, de acordo com a profundidade, textura, estrutura, porosidade e pedoforma, integradas entre si (Resende et al., 1997). De forma semelhan- te, observou-se, nos mapas, correspondência entre regiões de maior vazão com as áreas de maior recarga; as áreas de vegetação nativa também corresponderam às áreas de maior vazão, embora se verifique, em algumas situações, a correspondência de maior vazão com regiões de maior área de vegetação nativa e outras vezes com regiões de maior área de recarga (Figuras 4 e 5). Segundo Pinto (2003), a relação entre maiores vazões e a vegetação no entorno das nascentes ocorreu pela redução da velocidade do escoamento superficial promovida pela cobertura vegetal, permitindo maior permanência da água na superfície e, conseqüentemente, maior infiltração de água; Resende et al. (1997) também relataram haver infiltração deficiente de água nos locais com ausência de cobertura vegetal, seja pelo encrostamento provocado pelo impacto direto das gotas de chuva, seja pelas precipitações acentuadas que se concentram na superfície e escoam, ganhando energia e provocando erosão.

Portanto, com o uso de metodologias de análise geoestatística e de SIG, foi possível caracterizar regiões onde houve interação de variáveis geobiofísicas nas nascentes da bacia 

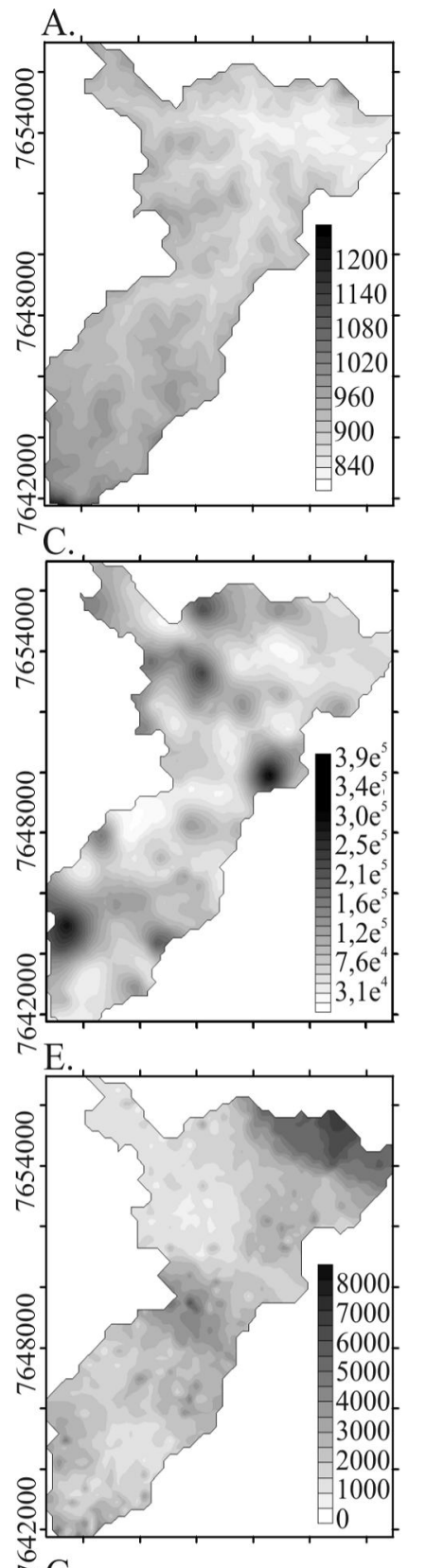

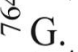

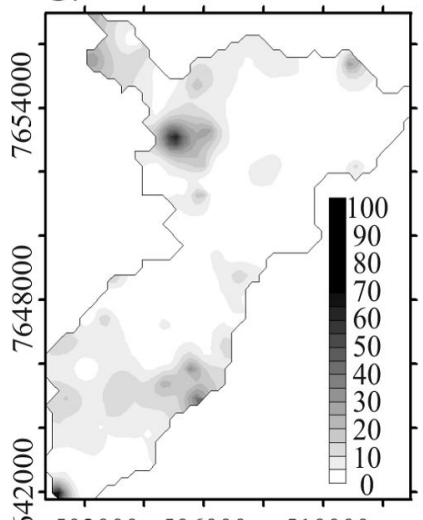

ऽ $502000506000 \quad 510000$
B.

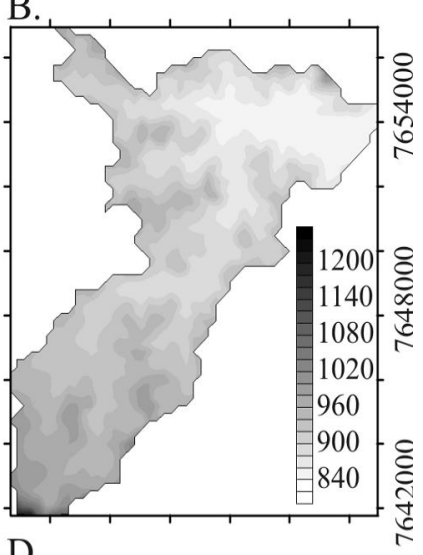

D.
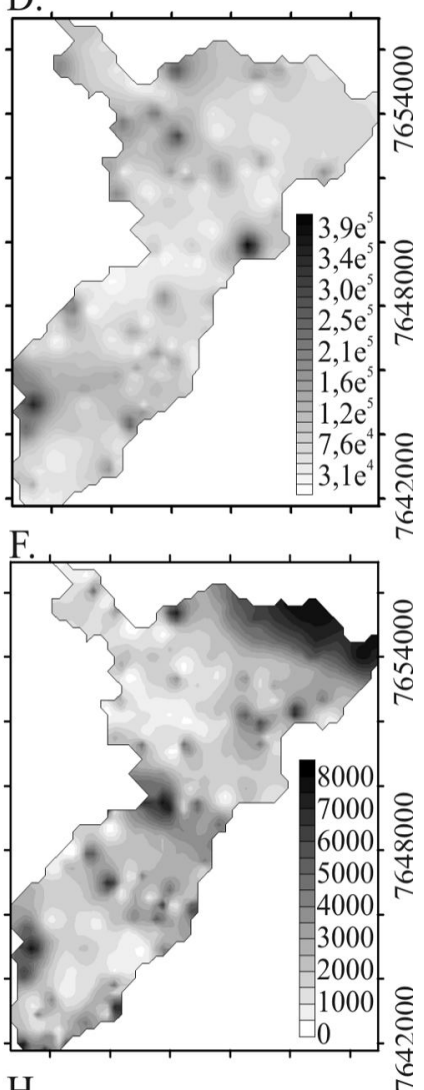

H.

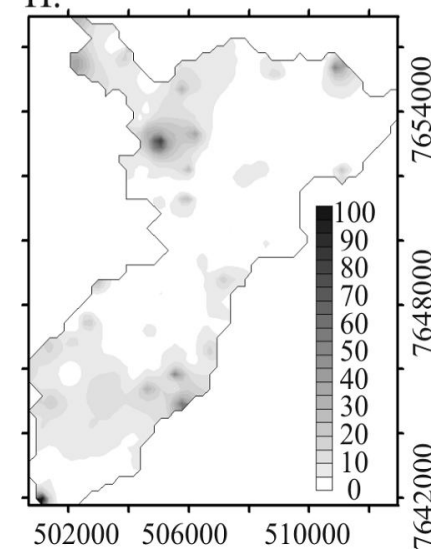

Figura 4. Representação bidimensional da interpolação por krigagem ordinária $(A, C, E, G)$ e por IPD $(B, D, F, H)$ referentes às variáveis: curva de nível $(A$, $B)$, área de recarga $\left(m^{2}\right)(C, D)$, área de vegetação nativa $\left(m^{2}\right)(E, F)$ e vazão $\left(\mathrm{L}\right.$ min $\left.^{-1}\right)$ das nascentes perenes $(G, H)$ da Bacia Hidrográfica do Ribeirão Santa Cruz, Lavras, M G
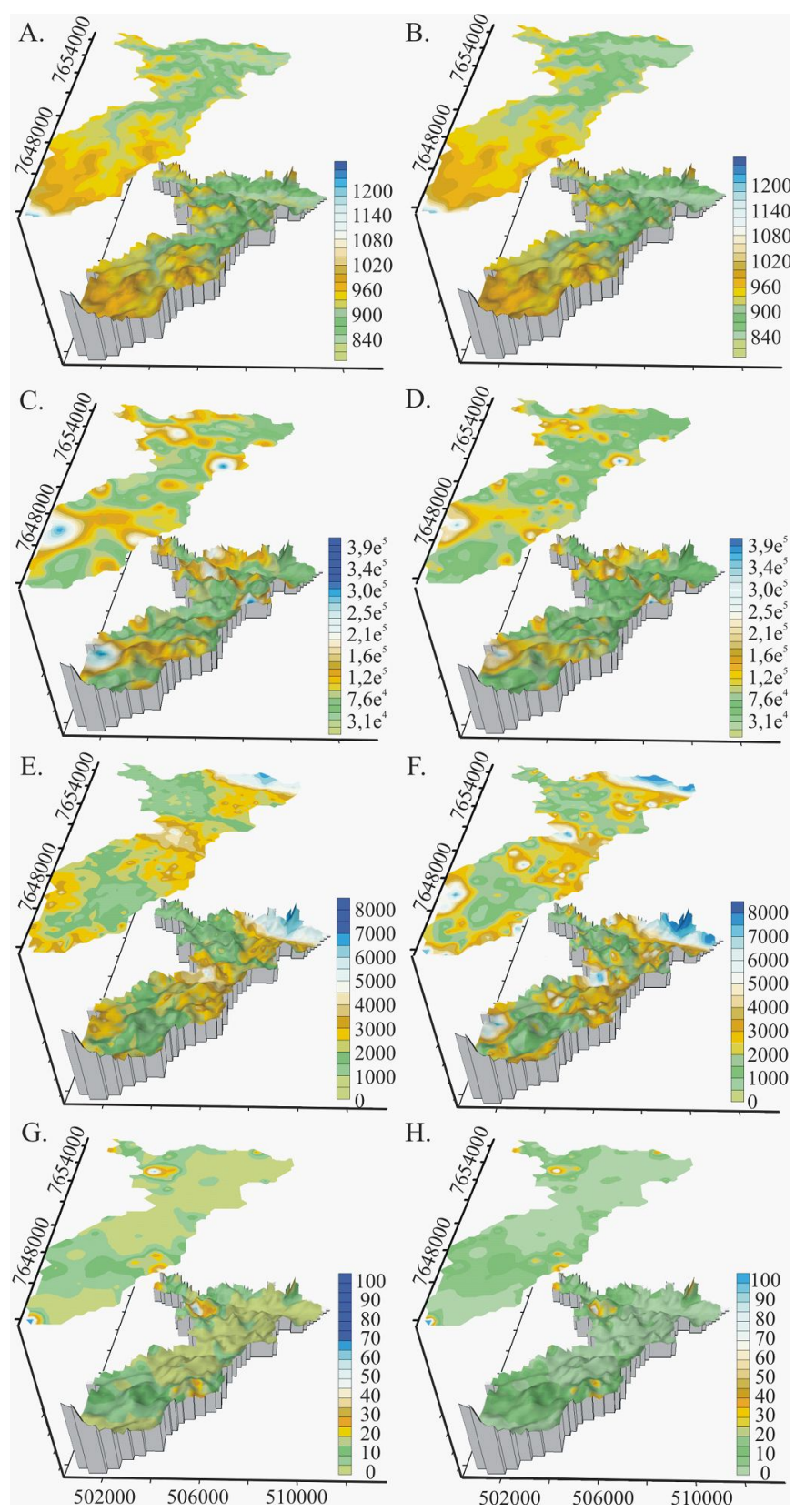

Figura 5. Representação da interpolação por krigagem ordinária $(A, C, E, G)$ e por IPD $(B, D, F, H)$ referentes às variáveis curva de nível $(A, B)$, área de recarga $\left(\mathrm{m}^{2}\right)(\mathrm{C}, \mathrm{D})$, área de vegetação nativa $\left(\mathrm{m}^{2}\right)(\mathrm{E}, \mathrm{F})$ e vazão $\left(\mathrm{Lmin}^{-1}\right)$ das nascentes perenes $(G, H)$, sobrepostas ao relevo da bacia hidrográfica do Ribeirão Santa Cruz, Lavras, M G

hidrográfica do Ribeirão Santa Cruz, bem como a distância máxima na qual as observações foram correlacionadas entre si, de acordo com a variabilidade, estrutura e magnitude de dependência espacial da altitude, vazão, vegetação nativa e área de recarga.

\section{CONCLUSÕES}

1. Por meio da Geoestatística e de Sistema de Informações Geográficas, foi possível representar e caracterizar a variabilidade espacial de variáveis geobiofísicas nas nascentes da Bacia Hidrográfica do Ribeirão Santa Cruz. 
2. Observou-se melhor qualidade das estimativas da krigagem ordinária quando comparadas ao IPD.

3. A preservação de uma nascente perene da bacia hidrográfica do Ribeirão Santa Cruz foi influenciada pela vizinhança em um raio próximo a $1620 \mathrm{~m}$, de acordo com o alcance obtido pelos semivariogramas dos dados de vazão e da área de recarga das nascentes.

\section{LITERATURA CITADA}

Alvarenga, M. I. N.; Paula, M. B. Planejamento conservacionista em microbacias. Informe Agropecuário, v.21, n.207, p.55-64. 2000.

Alves, S. C. A água como elemento fundamental da paisagem em microbacias. Informe Agropecuário, v.21, n.207, p.9-14. 2000.

Andrade, H.; Alves, H. M. R.; Vieira, T. G. C.; Esteves, D. R.; Resende, R. J. Diagnóstico ambiental do município de Lavras com base em dados georreferenciados do meio físico: IV-principais grupamentos de solos. In: Congresso Brasileiro de Engenharia Agrícola, 27, 1998, Poços de Caldas. Anais...Lavras: UFLA/SBEA, v.4, 1998, p.442-443.

Andriotti, J. L. S. Fundamentos de estatística e geoestatística. São Leopoldo: UNISINOS, 2003. 165p.

Bernardo, S. Manual de irrigação. 5.ed. Viçosa: UFV, Imprensa Universitária, 1989. 596p.

Boken, V. K.; Hoogenboom, G.; Hook, J. E.; Thomas, D. L.; Guerra, L. C.; Harrison, K. A. Agricultural water use estimation using geospatial modeling and a geographic information system. Agricultural Water Management, v.67, n.3, p.185-189, 2004.

Burrough, P. A.; McDonnell, R. A. Principles of geographical information systems. Oxford: Oxford University Press, 1998, 333p.

Camargo, E. C. G Desenvolvimento, implementação e teste de procedimentos geoestatísticos (krigeagem) no sistema de processamento de informações georreferenciadas (SPRING). São José dos Campos: INPE, 1997. 124p. Dissertação Mestrado

Cambardella, C. A.; Moorman, T. B.; Novak, J. M.; Parkin, T. B.; Karlen, D. L.; Turco, R. F.; Konopka, A. E. Fieldscale variability of soil properties in Central Iowa soils. Soil Science Society of America Journal, v.58, n.5, p.1501-1511, 1994.

Carvalho, J. R. P.; Vieira, S. R. Avaliação e comparação de estimadores de krigagem para variáveis agronômicas: Uma proposta. Campinas: Embrapa Informática Agropecuária, 2001. 21p.

Conde, R. P.; Yamamoto, J. K. Avaliação de reservas por métodos computacionais: um estudo de caso na mina de canoas 2 (PR). Revista Brasileira de Geociências, v.26, n.1, p.35-42. 1996.

Desbarats, A. J.; Logan, C. E.; Hinton, M. J.; Sharpe, D. R. On the kriging of water table elevations using collateral information from a digital elevation model. Journal of Hydrology, v.255, n.1, p.25-38. 2002.

Isaaks, E. H.; Srivastava, R. M. Applied geostatistics. New York: Oxford University Press, 1989. 561p.

Kravchenko, A. N. Influence of spatial structure on accuracy of interpolation Methods. Soil Science Society of America Journal, v.67, n.5, p.1564-1571, 2003.

Kravchenko, A.; Bullock, D. A comparative study of interpolation methods for mapping soil properties. Agronomy Journal, v.91, n.3, p.393-400, 1999.
Lombardi Neto, F. Manejo e conservação do solo. In: Lombardi Neto, F.; Camargo, O. A. (ed.) Microbacia do córrego São Joaquim (Município de Pirassununga, SP). Campinas: Instituto Agronômico, 1992. p.65-84.

Mueller, T. G.; Pierce, F. J.; Schabenberger, O.; Warncke, D. D. Map quality for site-specific fertility management. Soil Science Society of America Journal, v.65, n.5, p.1547-1558. 2001.

Musa, I. S.; Petterson, H. B. L.; Sivertun, A.; Lund, E. Spatial correlation between radon $(222 \mathrm{Rn})$ in groundwater and bedrock uranium (238U): GIS and geostatistical analyses. Journal of Spatial Hydrology, v.2, n.2, p.1-10, 2002.

Olea, R. A. Geostatistics for engineers and earth scientists. London: Kluwer Academic Publishers, 1999. 303p.

Pinto, L. V. A. Caracterização física da sub-bacia do Ribeirao Santa Cruz, Lavras, MG, e propostas de recuperação de suas nascentes. Lavras: UFLA, 2003. 165p. Dissertação Mestrado

Pinto, L. V. A.; Botelho, S. A.; Davide, A. C.; Ferreira, E. Estudo das nascentes da bacia hidrográfica do Ribeirão Santa Cruz, Lavras, MG. Scientia Forestalis, v.65, p.197-206, 2004.

Pinto, L. V. A.; Ferreira, E.; Botelho, S. A.; Davide, A. C. Caracterização física da bacia hidrográfica do Ribeirão Santa Cruz, Lavras, MG e uso conflitante da terra em suas áreas de preservação permanente. Cerne, v.11, n.1, p.49-60, 2005.

Resende, M.; Curi, N.; Rezende, S. B.; Corrêa, G. F. Pedologia: Base para distinção de ambientes. Lavras: UFLA, 1997. 367p.

Robertson, G. P. GS+: Geostatistics for the environmental sciences. Versão 5.03 Beta. Plainwell, Gamma Design Software, 1998. 152p.

Rocha, J. S. M. Manual de interpretação de aerofotogramas. 2.ed. Santa Maria: UFSM, 1985. 83p.

Salinger, M. J. Climate variability and change: Past, present and future - an overview, Climatic Change, v.70, n.1-2, p.9-29. 2005.

Shiklomanov, I. A. World water resources: A new appraisal and assessment for the 21st Century. Paris:UNESCO-IHP Publication, UNESCO, 1998. 37p.

Souza, E. R.; Fernandes, M. R. Sub-bacias hidrográficas: unidades básicas para o planejamento e a gestão sustentáveis das atividades rurais. Informe Agropecuário, v.21, n.207, p.15-20. 2000.

Surfer Version 8.0. Surface mapping system. Golden: Golden Software, 2002.

Vieira, S. R.; Maria, I. C.; Lombardi Neto, F.; Dechen, S. C. F.; Castro, O. M. Caracterização da variabilidade espacial de propriedades físicas. In: Lombardi Neto, F.; Camargo, O. A. (ed.) Microbacia do córrego São Joaquim (Município de Pirassununga, SP). Campinas: Instituto Agronômico, 1992. p.41-52.

Weber, D.; Englund, E. Evaluation and comparison of spatial interpolators II. Mathematical Geology, v.26, n.5, p.589-603. 1994.

Webster, R.; Oliver, M. Geostatistics for environmental scientists. England: John Wiley \& Sons, 2001. 271p.

Wollenhaupt, N. C.; Wolkowski, R. P.; Clayton, M. K. Mapping soil test phosphorus and potassium for variablerate fertilizer application. Journal of Production Agriculture,v.7, n.4, p.441-447, 1994.

Zimmerman, D.; Pavlik, C.; Ruggles, A.; Armstrong, M. P. An experimental comparison of ordinary and universal kriging and inverse distance weighting. Mathematical Geology, v.31, n.4, p.375-390, 1999. 